\title{
Analysis of Spurious Image Forces in Atomistic Simulations of Dislocations
}

\author{
B A Szajewski ${ }^{1}$ W A Curtin ${ }^{2}$ \\ ${ }^{1}$ School of Engineering, Brown University, Providence, RI, 02912 \\ ${ }^{2}$ Institute of Mechanical Engineering, EPFL, Lausanne, Switzerland 1015 \\ E-mail: Benjamin_Szajewski@brown.edu
}

\begin{abstract}
Molecular Dynamics simulations of dislocation/obstacle interactions are enhancing our physical understanding of plasticity. However, despite increasing computational power, the interaction between simulation cell boundaries and the long ranged fields of dislocations make spurious image effects inevitable. Here, these image effects are examined in detail, providing a general map of the spurious image stress as a function of simulation cell size, aspect ratio, and bow-out for both nominally edge and screw dislocations. This is achieved using an approximate image solution of the resulting boundary value problem as well as an analytic model that captures most of the spurious image effects. A unique simulation cell shape is found to minimize spurious image effects for a fixed simulation volume (i.e. fixed total number of atoms) and specified initial dislocation line length. The results are used to estimate image stress effects in various literature studies involving dislocation bow-out. The image effects are non-negligible. Several case studies involving simulation cell dimensions are shown to converge due to a near-zero scaling of the image stress with respect to the simulation cell dimensions used. Finally, a direct comparison is made between a dislocation bow-out configuration under an applied load in a finite simulation cell and an image-free multiscale simulation of the same problem and the difference is shown to be consistent with our estimated image stresses. Overall, the results here provide guidance for both the development and interpretation of quantitative Molecular Dynamics studies involving curved dislocation structures.
\end{abstract}

Keywords: Dislocation, Image Effects, Atomistic Simulation. 
This is an author-created, un-copyedited version of an article accepted for publication/published in Modelling And Simulation In Materials Science And Engineering. IOP Publishing Ltd is not responsible for any errors or omissions in this version of the manuscript or any version derived from it. The Version of Record is available online at http://dx.doi.org/10.1088/0965-0393/23/2/025008. Citation details: Szajewski, B. A.; Curtin, W. A. Modelling And Simulation In Materials Science And Engineering 2015, 23, .

\section{Introduction}

Fully atomistic methods have become an indispensible tool for providing direct physical insight into the mechanistic role of defects in materials [1-6]. Molecular statics simulations have been used extensively to study grain boundaries [7-9], dislocation cross-slip [10-12], crack tips [13, 14], and dislocation/obstacle interactions $[1-3,5,6,15-33]$. Due to the high computational cost, the size of simulation volumes (i.e. number of atoms) remains relatively small. For problems involving dislocations, which have long range stress and strain fields, the image forces associated with spurious interactions between dislocations and boundaries of the simulation cell can be nonnegligible, but have also been difficult to assess quantitatively. Nonetheless, various proposed methods to simulate dislocations [20-24] have proven useful in providing insight into plasticity phenomena. As computational materials science heads toward the goal of quantitative predictive results relevant to real materials, the issue of accuracy of the methods becomes more important. While one solution is to increase the simulation cell size, which is becoming more feasible as computational capabilities continue to expand, the need to perform many such simulations makes this only a partial solution. Thus, a quantitative understanding of spurious image interactions in standard MD simulations of dislocations is a useful benchmark for designing and interpreting simulation results. A variety of numerical methods have been developed to incorporate image forces based on finite element computations [34], and fourier-transform methods $[35,36]$ as well as exact analytic expressions for simple geometries [37, 38]. Here, we pursue a similar goal using other approaches and aimed at quantifying image forces within the standard simulation geometries used for simulating problems involving dislocation bow-out.

Since its inception [39] the Periodic Array of Dislocations (PAD) method [21-23, 40] has emerged, and gained acceptance over the past decade, as a means to model interactions between dislocations and other inhomogeneities. In this method, a single Volterra dislocation is inserted along the $x$ axis with its glide plane normal to the $z$ axis as shown in Fig. 1a. The simulation volume $\mathrm{V}=L_{x} \times L_{y} \times L_{z}$ has periodicity imposed both along the line $(x)$ and glide directions $(y)$, creating a periodic array of dislocations. Accomplishing periodicity along the glide direction for edge dislocations requires addition or removal of atoms, but various methods to do so are described in the literature $[6,22]$. The remaining out-of-plane direction is non-periodic, allowing for the application of stress [20] (or strain [22]). The initial singular dislocation structure is then relaxed using, e.g., the conjugate gradient method. The addition of solutes, precipitates, vacancies, or "quench methods" [41, 42] are then used to create obstacles to dislocation motion with the desired physical 
This is an author-created, un-copyedited version of an article accepted for publication/published in Modelling And Simulation In Materials Science And Engineering. IOP Publishing Ltd is not responsible for any errors or omissions in this version of the manuscript or any version derived from it. The Version of Record is available online at http://dx.doi.org/10.1088/0965-0393/23/2/025008. Citation details: Szajewski, B. A.; Curtin, W. A. Modelling And Simulation In Materials Science And Engineering 2015, 23, .

characteristics for the problem of interest. To apply a stress to the system using traction boundary conditions, the out-of-plane surfaces are subject to in-plane tractions by application of appropriate forces to atoms. For instance, to drive an edge dislocation using a traction boundary condition, the applied shear stress is $\tau_{y z}=F_{y} / A$ where $F_{y} / N$ represents the average force on each of the $N$ atoms acting over the upper or lower surface respectively (surface normal $z$ ) of area $A=L_{x} L_{y}$. Applied stresses can also be controlled by specifying displacement boundary conditions, i.e. fixing atoms on upper and lower surfaces, to generate the desired "applied" stress state in the interior of the system. Mixed boundary conditions (e.g. normal displacements and in-plane tractions) may also be used [6]. The defect-containing volume is then studied under varying applied loads. These simulations are typically carried out using a large-scale parallel atomistic simulation software such as LAMMPS [43].

Within the above scheme, the existence of spurious image forces due to the in-plane periodic images and the out-of-plane free or fixed surface are recognized, and attempts have been made to minimize their influence. Typically both $L_{y}$ and $L_{z}$ are made sufficiently large such that both the stacking fault width and the Peierls stress of a nominally straight dislocation converge to published values $[18,20,22,44]$. Satisfying such conditions is the minimum requirement, and does not consider image forces on non-straight dislocations. Thus, often, simulations are repeated with different simulation cell sizes to examine the reliability of the results. However, in many cases, simulations are performed for various periodic dislocation lengths (dimension $\left.L_{x}\right)$ so that the simulation cell dimensions, and corresponding image forces, are changing simultaneously with the problem of physical interest.

Here, we examine the spurious image forces arising in a typical dislocation bow-out simulation due to free out-of-plane surfaces and in-plane periodic images exclusively as a function of the normalized simulation cell volume $\left(\bar{V}=V / L_{x}^{3}\right)$, cell aspect ratio $\left(L_{y} / L_{z}\right)$, and the extent of bow-out of the dislocation. This is accomplished by direct calculation of the image forces using elasticity theory and a continuum representation of the dislocation and its periodic images. We also develop an analytic model that captures the majority of the spurious image forces. With these analyses, we provide a general map of the spurious forces on nominally edge and screw dislocations as a function of the simulation cell dimensions and the extent of dislocation bow-out. We find that the image forces act against the bow-out process, so the applied stress required to achieve any given level of bow-out is larger than in an image-free simulation. The total effect of the image field can be represented by an effective applied stress that counteracts the actual applied stress. We also make a comparison between a bowed-out dislocation 
This is an author-created, un-copyedited version of an article accepted for publication/published in Modelling And Simulation In Materials Science And Engineering. IOP Publishing Ltd is not responsible for any errors or omissions in this version of the manuscript or any version derived from it. The Version of Record is available online at http://dx.doi.org/10.1088/0965-0393/23/2/025008. Citation details: Szajewski, B. A.; Curtin, W. A. Modelling And Simulation In Materials Science And Engineering 2015, 23, .
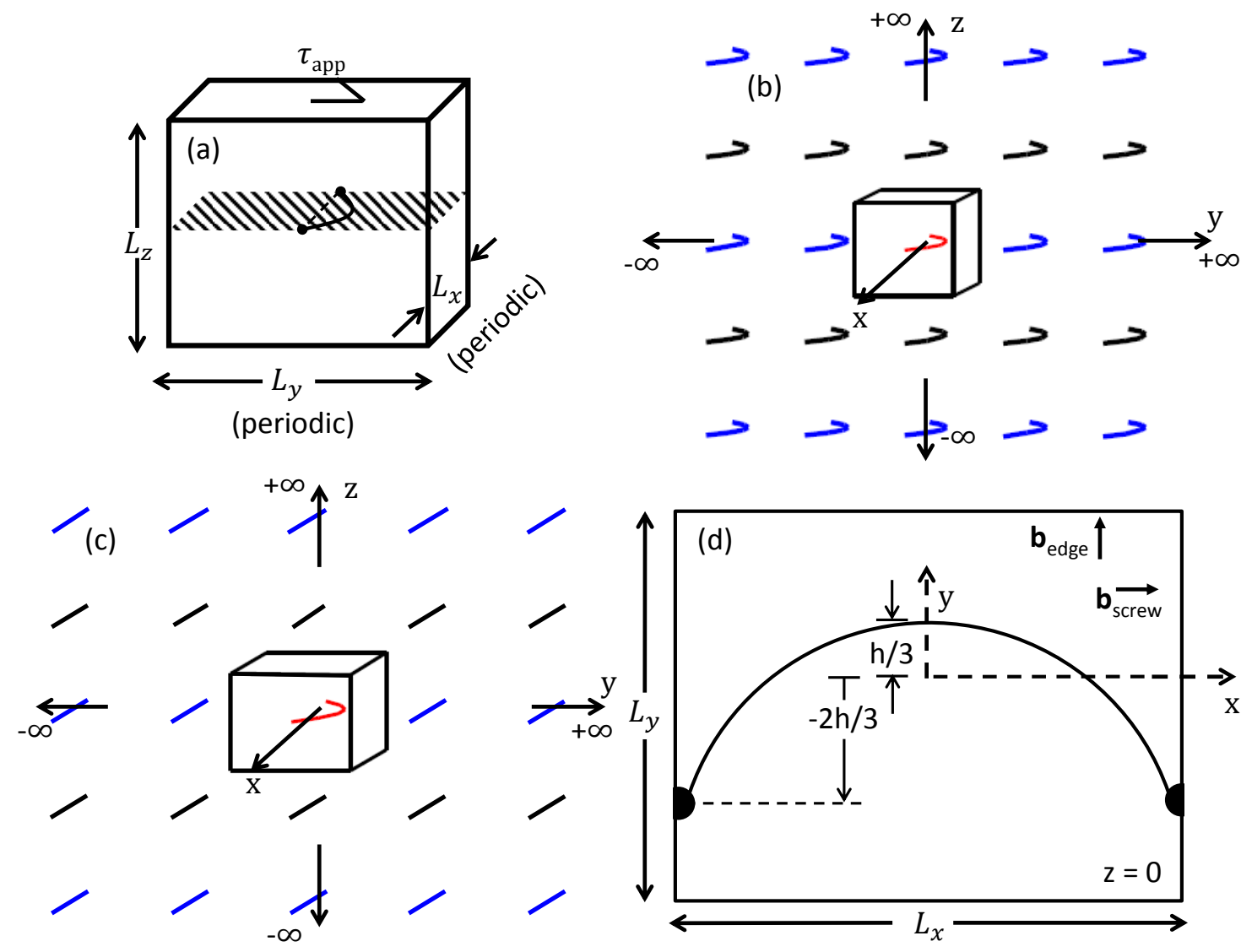

Figure 1. (a) Schematic view of the PAD simulation cell containing only the central dislocation; boundary conditions are imposed on the top and bottom surface, while all others surfaces are periodic. (b) Schematic view of the PAD simulation including only two sets of the infinite periodic and image dislocation array (red: primary dislocation along $x$, blue: images with positive Burgers vectors, black: images with negative Burgers vectors). (c) Schematic of the $2 d$ straight-image-dislocation approximation including only two sets of the infinite periodic and image dislocation array. (d) Top view of the periodic simulation cell depicting a bow-out of extent $h$ and the coordinate system used here with the origin centered at $2 \mathrm{~h} / 3$ ahead of the pinning points for a dislocation with maximum bow-out $h$. 
This is an author-created, un-copyedited version of an article accepted for publication/published in Modelling And Simulation In Materials Science And Engineering. IOP Publishing Ltd is not responsible for any errors or omissions in this version of the manuscript or any version derived from it. The Version of Record is available online at http://dx.doi.org/10.1088/0965-0393/23/2/025008. Citation details: Szajewski, B. A.; Curtin, W. A. Modelling And Simulation In Materials Science And Engineering 2015, 23, .

in a finite-size simulation cell and in an image-free multiscale simulation [45] and show the difference to be consistent with our computed effective applied stress due to the image forces. We then extend this comparison to several other studies reported in the literature. Our analyses and results provide guidance for estimating image forces in any given PAD simulation or, conversely, for choosing simulation cell sizes and shapes to minimize spurious image forces.

\section{Methods to Compute Image Forces}

The well-known Peach-Koehler equation (e.g. [46]) relates the stress fields $(\boldsymbol{\sigma})$ to the driving force $(\mathbf{F})$ acting on a segment of dislocation $\left(d \mathbf{l}^{\prime}\right)$. By projecting this force on to the slip plane (unit normal $\mathbf{n}$ ) with Burgers vector b,

$$
\mathbf{F} \cdot\left(\mathbf{n} \times \mathrm{dl}^{\prime}\right) /\left|\mathrm{dl}^{\prime}\right|^{2}=(\boldsymbol{\sigma} \mathbf{b} \cdot \mathbf{n})
$$

the component(s) of stress responsible for glide are obtained. Our goal here is to determine the spurious image stresses, and thus the corresponding spurious Peach-Koehler forces, for the simple problem of a dislocation bowing out around periodically spaced pinning points when simulated by Molecular Statics using the PAD geometry with traction boundary conditions. The geometry of the problem is shown in Fig. 1a. We assume a bowed-out dislocation configuration corresponding to an arc with a constant radius of curvature terminating at pinning points on the boundaries of the cell along the $x$ direction, so that in the absence of bow-out the nominal dislocation length is $L_{x}$. Fig. 1b shows the "image" dislocations that partially satisfy the periodic and free-surface boundary conditions of the PAD simulation cell. We denote $\tau_{i m g}^{\text {edge }}=\left.\sigma_{y z}\right|_{x, y, z=0}$ and $\tau_{i m g}^{\text {screw }}=\left.\sigma_{x z}\right|_{x, y, z=0}$ as the spurious image stress fields at positions $(\mathrm{x}, \mathrm{y}, 0)$ along the $(\mathrm{x}, \mathrm{y}, 0)$ line of the central curved dislocation that arise from the imposed boundary conditions of the (traction) PAD method. Our goal is to compute these image stresses as a function of simulation cell size, cell shape, and dislocation bow-out (curvature). There are three methods to obtain these image stresses. The first method involves solving the posed boundary value problem using a numerical solution via fourier transform methods or Finite Element Methods (FEM) similar to those used in continuum discrete dislocation modeling [35, 47]; both the FEM and fourier transform procedures are general but pose several challenges such as implementation of periodic boundary conditions, computational cost, and complexity, while lacking direct insight and thus are not pursued here. The second method is a direct computation using an infinite 
This is an author-created, un-copyedited version of an article accepted for publication/published in Modelling And Simulation In Materials Science And Engineering. IOP Publishing Ltd is not responsible for any errors or omissions in this version of the manuscript or any version derived from it. The Version of Record is available online at http://dx.doi.org/10.1088/0965-0393/23/2/025008. Citation details: Szajewski, B. A.; Curtin, W. A. Modelling And Simulation In Materials Science And Engineering 2015, 23, .

array of curved image dislocations, as indicated in Fig. 1b, so as to satisfy the imposed shear boundary conditions. The third method involves an additional approximation leading to analytic expressions in which the curved image dislocations are replaced by straight dislocations at appropriate positions. For edge dislocations, both the second and third methods require an additional superposed Airy stress function to nullify the normal stresses present on the upper and lower surfaces. We discuss and present here both the second and third methods.

\subsection{Curved Image Dislocations}

Our objective is to determine the driving shear stress imposed on a (curved) dislocation configuration with periodicity in the $x$ and $y$ directions confined to the central slip plane, by the boundary conditions $\left.\mathbf{t}\right|_{z=+/-\frac{L_{z}}{2}}=\mathbf{0}$. We start by examining two features of existing analytic solutions for the limiting case of a simulation containing only a single, straight, infinite dislocation aligned with the $\mathrm{x}$-axis in an infinite plate, as proposed by Chou [48] for the screw and Nabarro [49] for the edge. The premise of these solutions involves the superposition of an infinite array of dislocations with alternating Burgers vectors along the out-of-plane direction and an additional Airy stress function for the edge. This scheme is directly extensible to finite dislocation segments and is demonstrated as follows. The stress tensor field at point $(\mathrm{x}, \mathrm{y}, \mathrm{z})$ generated by a dislocation segment is $[46]$

$$
\begin{aligned}
\boldsymbol{\sigma} & =\frac{\mu}{4 \pi} \oint\left(\mathbf{b} \times \nabla^{\prime}\right) \frac{1}{R} \otimes \mathrm{d}^{\prime} \\
& +\frac{\mu}{4 \pi} \oint \mathrm{d} \mathbf{l}^{\prime} \otimes\left(\mathbf{b} \times \nabla^{\prime}\right) \frac{1}{R} \\
& -\frac{\mu}{4 \pi} \oint \frac{\nabla^{\prime} \cdot\left(\mathbf{b} \times \mathrm{d} \mathbf{l}^{\prime}\right)\left(\nabla \otimes \nabla-\mathbf{I} \nabla^{2}\right) R}{(1-\nu)}
\end{aligned}
$$

where $\mu$ is the shear modulus, $R=\sqrt{\left(x^{\prime}-x\right)^{2}+\left(y^{\prime}-y\right)^{2}+\left(z^{\prime}-z\right)^{2}}$ and the integrals are taken along the dislocation segment $\left(\mathrm{dl}^{\prime}\right)$ with respect to a field point, $\mathbf{x}=[x, y, z]$. Defining an origin on a free surface (unit normal $\mathbf{n}$ oriented along the $\mathrm{z}$ direction), two shear stress terms $\left(\sigma_{x z}, \sigma_{y z}\right)$ and a normal stress $\left(\sigma_{z z}\right)$ arise due to the presence of a dislocation segment having line direction and Burgers vector perpendicular to $\mathbf{n}$, i.e. 
This is an author-created, un-copyedited version of an article accepted for publication/published in Modelling And Simulation In Materials Science And Engineering. IOP Publishing Ltd is not responsible for any errors or omissions in this version of the manuscript or any version derived from it. The Version of Record is available online at http://dx.doi.org/10.1088/0965-0393/23/2/025008. Citation details: Szajewski, B. A.; Curtin, W. A. Modelling And Simulation In Materials Science And Engineering 2015, 23, .

$$
\begin{aligned}
\sigma_{\alpha z} & =\frac{\mu}{4 \pi} \oint \frac{\mathbf{n} \cdot(\Delta \mathbf{x} \times \mathbf{b}) \mathrm{d} x_{\alpha}^{\prime}}{R^{3}} \\
& +\frac{\mu}{4 \pi} \oint \frac{\Delta x_{\alpha}\left(R^{2}-3 z^{\prime 2}\right) \mathbf{n} \cdot\left(\mathbf{b} \times \mathrm{d} \mathbf{l}^{\prime}\right)}{(1-\nu) R^{5}}
\end{aligned}
$$

and

$$
\sigma_{z z}=\frac{\mu}{4 \pi} \oint \frac{\left(z^{\prime}\right)\left(R^{2}-3 z^{\prime 2}\right) \mathbf{n} \cdot\left(\mathbf{b} \times \mathrm{d} \mathbf{l}^{\prime}\right)}{(1-\nu) R^{5}}
$$

where $\Delta \mathbf{x}=\left[\left(x^{\prime}-x\right),\left(y^{\prime}-y\right),\left(z^{\prime}-z\right)\right]$ and $\alpha=x, y$.

The shear stresses, Eq. (3), are odd in $\mathbf{b}$ and independent of the side of the plane on which the segment resides (i.e. independent of $\operatorname{sgn}\left(z^{\prime}\right)$ ). To annul the shear tractions on both upper and lower surfaces of the plate geometry, we therefore proceed in a similar manner to Chou [48] by superposing alternating signed dislocation segments outside of the PAD region. Specifically (referring to Fig. 1b) for a given dislocation segment within the PAD described by Eqs. (3) and (4) with stress fields $\boldsymbol{\sigma}(+\mathbf{b}), 2 \mathrm{~N}$ corresponding segments are placed at distances $z=+/-i L_{z}$ with stress fields $\boldsymbol{\sigma}\left((-1)^{i} \mathbf{b}\right)$ and $i=1,2,3, \ldots N$. Within this superposition scheme the resolved shear tractions on planes $z=+/-\frac{L_{z}}{2}$ as $N \rightarrow \infty$ are $\mathbf{0}$.

Second we note that the normal stress, Eq. (4), is both odd in $\mathbf{b}$ and $z^{\prime}$ and therefore $\left(\sigma_{z z}\right)$ is not necessarily zero on either free surface in the plate geometry when using the above image construction. The plate solution presented by Nabarro [49] edge dislocation uses an Airy stress function $\left(\chi^{s}(y, z)\right)$ to annul these surface normal stresses while producing an additional shear stress on the slip plane. To approximately annul the normal stress, Eq. (4), in our problem involving curved dislocations, we include the same Airy stress function as proposed by Nabarro [49] for the straight edge dislocation placed at the origin along the center-of-mass line (in the y-direction) of the curved dislocation structure, as indicated in Figs. 1c,d. For the circular arc shape considered here, the peak of the bow-out and the center-of-mass position of the curved dislocation are at distances $h$ and $2 h / 3$ ahead of the initial dislocation line, respectively. The origin of the Airy stress function therefore evolves with the bow-out configuration (with increasing $\mathrm{h}$ ).

The periodicity along the glide direction $y$ of the PAD method is then achieved by superimposing a periodic array of the solutions above for the single dislocation in the infinite plate problem, including the superposition of the Airy stress function evaluated at the center-of-mass site of each (edge) dislocation. Since each vertical array plus Airy correction satisfies the desired boundary conditions, the periodic sum over images in the $y$ direction satisfies 
This is an author-created, un-copyedited version of an article accepted for publication/published in Modelling And Simulation In Materials Science And Engineering. IOP Publishing Ltd is not responsible for any errors or omissions in this version of the manuscript or any version derived from it. The Version of Record is available online at http://dx.doi.org/10.1088/0965-0393/23/2/025008. Citation details: Szajewski, B. A.; Curtin, W. A. Modelling And Simulation In Materials Science And Engineering 2015, 23, .

the desired boundary conditions automatically. For the Airy stress function term, the summation is

$$
\tau_{\text {img }, \text { Airy }}^{\text {edge }}(y)=\sum_{i=-N}^{N}-\left.\left(\frac{\partial^{2} \chi^{s}(y, z)}{\partial y \partial z}\right)\right|_{y-i L_{y}, z=0}
$$

This discrete sum over $i$ can be simplified using both the angle sum and Lagrange trig identities resulting in total additional shear stress on the slip plane for a nominally edge dislocation given by

$$
\frac{\tau_{\text {img }, \text { Airy }}^{\text {edge }}(y) L_{y}}{\mu b}=\frac{L_{y}}{L_{z}} \int_{0}^{\infty} \frac{n^{2} \sin \left(2 n \frac{y}{L_{y}} \frac{L_{y}}{L_{z}}\right) \tanh ^{2}(n) \sin \left((2 N+1)\left(n L_{y} / L_{z}\right)\right)}{\pi(1-\nu)(n-\cosh (n) \sinh (n)) \sin \left(n L_{y} / L_{z}\right)} \mathrm{d} n
$$

In the limit that $N \rightarrow 0$ this additional shear stress term reduces to the result given by Nabarro [49] for the single edge dislocation in an infinite plate.

To implement the above curved image construction, we create the corresponding image dislocations in all three dimensions similar to Fig. 1b, but extending out to only a finite number of images $(\mathrm{N}=25)$ in each direction, and then add the shear stress generated by the modified periodic Airy stress function Eq. (6) for the nominally edge dislocation. Each curved image dislocation is then represented by a discrete set of straight dislocation segments, i.e. discretized in exactly the same manner as is commonly used in various discrete dislocation simulations. The modified periodic Airy stress function is evaluated numerically using the "integral" function within Matlab. When the central dislocation is included, the sum over an infinite number of images produces nearly zero tractions on both free surfaces of the simulation cell. To obtain the image forces on the central dislocation, we sum the stress fields of the finite set of discretized image dislocations along the line of the central dislocation. This is similar to nodal force calculations performed within 3d discrete dislocation codes such as ParaDis [50] but we have written a separate code within Matlab to obtain the results presented here.

For data presented below in Figs. 2 and 3, we have used 25 periodic images of the central dislocation in all three directions $\mathrm{x}, \mathrm{y}$, and $\mathrm{z}$ with 5 segments per image. We have performed similar calculations using only 13 images in all directions and also using a small finite-sized obstacle rather than a point obstacle in pinning the dislocation. The differences in results are negligible. The finite size of our image array leads to shear stresses on the out-of-plane surfaces of at most $2 \mathrm{MPa}$, so that these surfaces are not truly traction free over the entire surface area, and this error decreases with 
This is an author-created, un-copyedited version of an article accepted for publication/published in Modelling And Simulation In Materials Science And Engineering. IOP Publishing Ltd is not responsible for any errors or omissions in this version of the manuscript or any version derived from it. The Version of Record is available online at http://dx.doi.org/10.1088/0965-0393/23/2/025008. Citation details: Szajewski, B. A.; Curtin, W. A. Modelling And Simulation In Materials Science And Engineering 2015, 23, .

increasing images as expected. Correcting for these stresses would lead to image stresses on the central dislocation that differ from our results by less than $2 \mathrm{MPa}$. Using the finite image array, we then calculate the driving stress $\tau_{y z}$ or $\tau_{x z}$ along the line of the curved central dislocation. We repeat this type of calculation for a range of simulation cell dimensions and magnitudes of dislocation bow-out for both nominally edge and screw dislocations.

\subsection{Approximation using Straight Image Dislocations}

Numerical computations of the image stresses of the curved dislocations can be performed but do not provide significant insights. Since the image dislocations are not usually too close to the central dislocation, the role of their curvature is decreased as the simulation cell size is increased. We therefore consider an image array where the curved image dislocations are replaced by a set of straight image dislocations, shown in Fig. 1c. This approximation allows for analytic estimates of the image stresses on the central curved dislocation. We account for the bow-out of the central curved dislocation by using a coordinate system for the image dislocations identical to that described in section 2.1 for the Airy stress function, where the origin is located at the center-of-mass line of the central bow-out ahead of the initial dislocation position, as indicated in Fig. 1d. For a dislocation bowing out in to a circular arc, the center-of-mass position is very accurately located at $2 h / 3$ ahead of the initial position, almost independent (within $2 \%$ ) of the amount of bow-out $h / L_{x}$. Specifically, we define an $(\mathrm{x}, \mathrm{y}, \mathrm{z})$ coordinate system with the $x$ axis lying along the position $2 h / 3$ of the bow-out, so that the actual curved dislocation line ranges from the positions $\left(-L_{x} / 2,-2 h / 3\right)$ and $\left(L_{x} / 2,-2 h / 3\right)$ at the pinning points to $(0, h / 3)$ at the point of greatest bow-out. The straight image dislocations then translate with the evolving bow-out in h, and always replicate the mean burgers vector distribution of the curved dislocation structure. This simplification neglects dipolar dislocation segments with spacing and length on the order of $L_{x}$ and $h$, leading to an error in the stress scaling as $\sim \mathcal{O}\left(\frac{b h L_{x}}{L_{y}^{3}}\right)$.

The image stress at position $(\mathrm{x}, \mathrm{y}, 0)$ in the plane of the central dislocation is then given by the sum of the stresses due to the 2-dimensional array of straight image dislocations and the modified periodic Airy stress function for the straight edge dislocation. The stress field of a 1-dimensional array of infinite straight edge dislocations aligned along the $x$ axis and with periodicity $L_{y}$ is given by [46],

$$
\frac{\sigma_{y z}^{1 d-e d g e}(Y, Z) L_{y}}{\mu b}=\frac{\sin (Y)(\cosh (Z)-\cos (Y)-Z \sinh (Z))}{2(1-\nu)(\cosh (Z)-\cos (Y))^{2}}
$$


This is an author-created, un-copyedited version of an article accepted for publication/published in Modelling And Simulation In Materials Science And Engineering. IOP Publishing Ltd is not responsible for any errors or omissions in this version of the manuscript or any version derived from it. The Version of Record is available online at http://dx.doi.org/10.1088/0965-0393/23/2/025008. Citation details: Szajewski, B. A.; Curtin, W. A. Modelling And Simulation In Materials Science And Engineering 2015, 23, .

and similarly for the screw

$$
\frac{\sigma_{x z}^{1 d-\text { screw }}(Y, Z) L_{y}}{\mu b}=\frac{\sin (Y)}{2(\cosh (Z)-\cos (Y))}
$$

where $Y(y)=2 \pi y / L_{y}$ and $Z(z)=2 \pi z / L_{y}$. For a 2 -d periodic array with spacing $L_{z}$ and alternating Burgers vectors, the stress field is obtained by shifting the 1-d solution by integer units of $L_{z}$, alternating the sign of the Burgers vector, and summing over all the images, leading to

$$
\sigma_{y z}^{2 d-e d g e}(y, z)=\sum_{n=-\infty}^{+\infty}(-1)^{n} \sigma_{y z}^{1 d-e d g e}\left(Y, Z\left(z-n L_{z}\right)\right)
$$

and

$$
\sigma_{x z}^{2 d-\text { screw }}(y, z)=\sum_{n=-\infty}^{+\infty}(-1)^{n} \sigma_{x z}^{1 d-\text { screw }}\left(Y, Z\left(z-n L_{z}\right)\right)
$$

We note the work of Cai and coworkers $[51,52]$ on conditional convergence issues related to these (2D) summations. In the most general case, a linear term and a constant spurious term arise due to the $\sim 1 / R$ stress fields being summed over a $2 \mathrm{~d}(\sim R d R)$ domain. For these two specific cases (eq. (8a) and $(8 b))$, it has been demonstrated [51] that the correction terms are zero, and hence absolute convergence is possible.

The image stresses should not include the stress due to the central dislocation itself, and so the approximate image stress fields in the $(\mathrm{x}, \mathrm{y}, \mathrm{z}=0)$ plane are

$$
\tau_{i m g}^{e d g e}(y)=\tau_{i m g, A i r y}^{e d g e}(y)+\left.\sigma_{y z}^{2 d-e d g e}\right|_{z=0}-\frac{\mu b}{2 \pi y(1-\nu)}
$$

and

$$
\tau_{\text {img }}^{\text {screw }}(y)=\left.\sigma_{x z}^{2 d-s c r e w}\right|_{z=0}-\frac{\mu b}{2 \pi y}
$$

which depend on the 2-d aspect ratio $L_{y} / L_{z}$. We numerically evaluate Eqs. (9a), and (9b) for a range of aspect ratios and for positions $\mathrm{y}(\mathrm{x})$ along the line of the bowed out dislocation $\left(-L_{x} / 2<x<L_{x} / 2\right)$. We truncate the infinite summation at $|n| \leq 1001$ leading to $<0.1 \%$ error in the shear tractions on the upper and lower surfaces.

\subsection{Analytic Scaling of Image Forces and an Effective Image Stress}

To gain insight into the scaling of the image stress fields with respect to both the cell dimensions and bow-out, we analyze the results of Sec. 2.2 
This is an author-created, un-copyedited version of an article accepted for publication/published in Modelling And Simulation In Materials Science And Engineering. IOP Publishing Ltd is not responsible for any errors or omissions in this version of the manuscript or any version derived from it. The Version of Record is available online at http://dx.doi.org/10.1088/0965-0393/23/2/025008. Citation details: Szajewski, B. A.; Curtin, W. A. Modelling And Simulation In Materials Science And Engineering 2015, 23, .

further. Recognizing that $y / L_{y}$ is small because $|y| \leq h$ and $h \leq L_{y}$ (see Fig. 1d), we expand (9a) and (9b) to first order in $y / L_{y}$. Defining the out-of-plane aspect ratio as $\bar{L}=L_{y} / L_{z}$, the image stress at the position $y(x)$ along the curved dislocation can be written as

$$
\frac{\tau_{i m g}^{\text {edge }}[y(x)] L_{y}}{\mu b}=\left[\bar{L}^{2} A(\bar{L})+B(\bar{L})\right]\left(\frac{y(x)}{L_{y}}\right)
$$

and

$$
\frac{\tau_{\text {img }}^{\text {screw }}[y(x)] L_{y}}{\mu b}=C(\bar{L})\left(\frac{y(x)}{L_{y}}\right)
$$

where

$$
\begin{aligned}
A(\bar{L}) & =\frac{2}{\pi(1-\nu)} \int_{0}^{\infty} \frac{n^{3} \tanh ^{2}(n) \sin ((2 N+1)(n \bar{L}))}{(n-\cosh (n) \sinh (n)) \sin (n \bar{L})} \mathrm{d} n \\
B(\bar{L}) & =\frac{\pi}{(1-\nu)}\left[\sum_{n=1}^{\infty}(-1)^{n} \operatorname{csch}^{2}\left(\frac{n \pi}{\bar{L}}\right)\left(1-\frac{2 n \pi}{\bar{L}} \operatorname{coth}\left(\frac{n \pi}{\bar{L}}\right)\right)-\frac{1}{6}\right] \\
C(\bar{L}) & =2 \pi\left[\sum_{n=1}^{\infty} \frac{(-1)^{n}}{\cosh \left(\frac{2 \pi n}{\bar{L}}\right)-1}-\frac{1}{12}\right]
\end{aligned}
$$

This approximate image stress field thus scales directly with the distance $y$ from the origin, with $-2 h / 3<y<h / 3$, and has no explicit dependence on $L_{x}$, as expected. Several further manipulations can then be made. First, a general reference stress for dislocation bow-out problems is the Frank-Read source operation stress, which scales as $\tau_{F R} \sim \frac{\mu b}{L_{x}}$. Second, since the cost of molecular simulations scales directly with the volume of the simulation cell, we define a normalized volume $\bar{V}=L_{y} L_{z} / L_{x}^{2}$. Introducing these quantities into Eqs. (10a) and (10b), we obtain the image stress fields in terms of $\bar{L}$ and $\bar{V}$ as

$$
\begin{gathered}
\tau_{\text {img }}^{\text {edge }}[y(x)]=\left(\frac{\mu b}{L_{x}}\right)\left(\frac{y(x)}{L_{x}}\right) \bar{\tau}_{\text {img }}^{\text {edge }}(\bar{L}, \bar{V}) \\
\tau_{\text {img }}^{\text {screw }}[y(x)]=\left(\frac{\mu b}{L_{x}}\right)\left(\frac{y(x)}{L_{x}}\right) \bar{\tau}_{\text {img }}^{\text {screw }}(\bar{L}, \bar{V})
\end{gathered}
$$

where we have defined the dimensionless image stress gradient as

$$
\bar{\tau}_{i m g}^{e d g e}(\bar{L}, \bar{V})=\frac{\bar{L}^{2} A(\bar{L})+B(\bar{L})}{\bar{V} \bar{L}}
$$

and

$$
\bar{\tau}_{\text {img }}^{\text {screw }}(\bar{L}, \bar{V})=\frac{C(\bar{L})}{\bar{V} \bar{L}}
$$


This is an author-created, un-copyedited version of an article accepted for publication/published in Modelling And Simulation In Materials Science And Engineering. IOP Publishing Ltd is not responsible for any errors or omissions in this version of the manuscript or any version derived from it. The Version of Record is available online at http://dx.doi.org/10.1088/0965-0393/23/2/025008. Citation details: Szajewski, B. A.; Curtin, W. A. Modelling And Simulation In Materials Science And Engineering 2015, 23, .

While the image stress fields along the line of the dislocation (Eqs. (11a) and $(11 b))$ provide insight, they are not directly interpretable relative to the true applied stress and the nominally applied stress in a PAD simulation. Consequently, we now derive an effective image stress, $\tau_{\text {img,eff }}$, that is (i) spatially constant, that (ii) represents the net effect of the image forces on the bowing dislocation, and that can be (iii) directly subtracted from the nominally applied stress in a PAD simulation. The effective image stress $\tau_{\text {img,eff }}$ is determined as a net configurational force acting on the bowing out dislocation in the following manner. Imagine a dislocation bowed out into an arc of a circle with maximum bow-out $h$ and consider an incremental increase in both the bow-out and the $2 \mathrm{~d}$ image array so that the maximum bow-out is $h+\delta h$ while the image array translates by $\frac{2}{3} \delta h$. We denote the incremental area swept out as $\delta A$. Through this process, the image stress field $\tau_{\text {img }}[y(x)]$ acts along the arc of the dislocation, in some places pushing the dislocation forward and in places pushing it back. The total incremental work done by the image stresses in making the incremental motion $(\mathrm{d} A=A(h+\delta h)-A(h))$ is

$$
\delta W=\oiint_{A} \tau_{i m g}[y(x)] b \mathrm{~d} A
$$

Using the analytic expressions for $\tau_{i m g}[y(x)]$ in Eq. 11a or b, the work can be computed to first order in $h / L_{x}$ as

$$
\delta W \approx\left(\frac{\mu b^{2}}{L_{x}}\right)\left(\frac{4 h}{45 L_{x}}\right) \bar{\tau}_{i m g}(\bar{L}, \bar{V}) \delta A
$$

for the edge and similarly for the screw. The net incremental work when including the effective image stress acting over the same incremental area however must be zero, so

$$
\oiiint_{A} \tau_{i m g, e f f} b \mathrm{~d} A=-\delta W
$$

and hence our final estimate for the effective image stress is

$$
\tau_{\text {img,eff }}^{\text {edge }} \approx-\left(\frac{\mu b}{L_{x}}\right)\left(\frac{4 h}{45 L_{x}}\right) \bar{\tau}_{\text {img }}^{\text {edge }}(\bar{L}, \bar{V})
$$

and

$$
\tau_{\text {img,eff }}^{\text {screw }} \approx-\left(\frac{\mu b}{L_{x}}\right)\left(\frac{4 h}{45 L_{x}}\right) \bar{\tau}_{i m g}^{\text {screw }}(\bar{L}, \bar{V})
$$


This is an author-created, un-copyedited version of an article accepted for publication/published in Modelling And Simulation In Materials Science And Engineering. IOP Publishing Ltd is not responsible for any errors or omissions in this version of the manuscript or any version derived from it. The Version of Record is available online at http://dx.doi.org/10.1088/0965-0393/23/2/025008. Citation details: Szajewski, B. A.; Curtin, W. A. Modelling And Simulation In Materials Science And Engineering 2015, 23, .

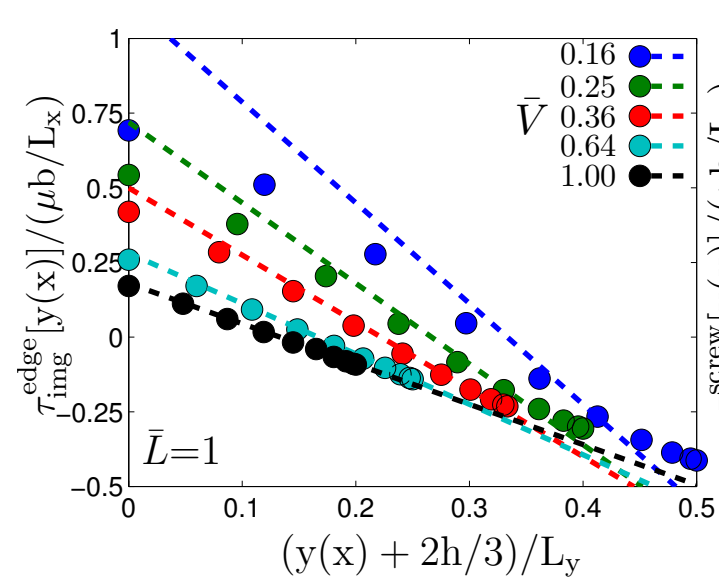

(a) Edge

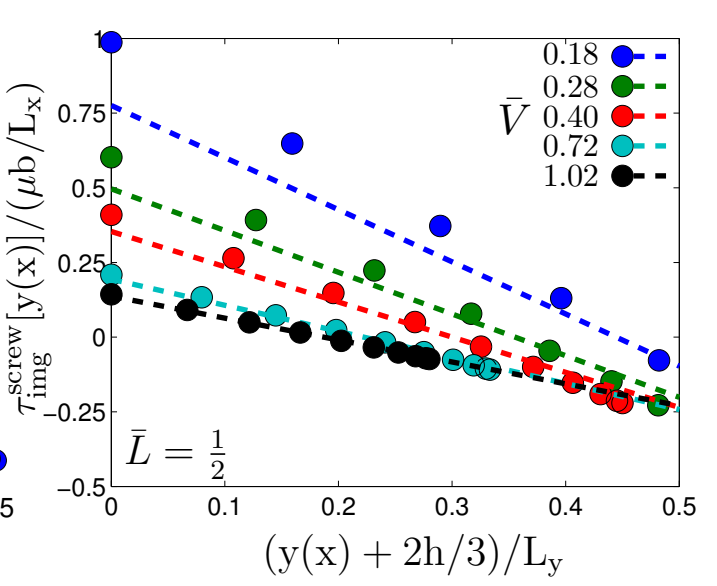

(b) Screw

Figure 2. (a) Edge and (b) Screw. Image stress field, normalized by the Frank-Read stress $\left(\mu b / L_{x}\right)$, vs. normalized position along the (curved) dislocation line for several normalized volumes $\left(\bar{V}=L_{y} L_{z} / L_{x}^{2}\right)$ and $h=0.2 L_{x}$, as predicted by the $3 \mathrm{~d}$ discrete analysis (symbols) and by the $2 \mathrm{~d}$ model (dashed lines). The curved dislocation spans the range from the pinning point, $y(x)=-2 h / 3$ (and hence $(y(x)+2 h / 3) / L y=0)$, to the peak $y(0)=h / 3$ (and hence $(y(x)+2 h / 3) / L y=h / L y)$. For the cases $\mathrm{h}=0.2 \mathrm{Lx}$ shown here, the peak is then at $0.2(L x / L y)=0.2 / \sqrt{\bar{L} \bar{V}}$.

\section{Results}

We start by comparing the results of the $3 \mathrm{~d}$ curved image array and the $2 \mathrm{~d}$ straight image approximation, (11a) and (11b). The independent variables which determine the image stress field are the two characteristic simulation cell dimensions $\bar{L}$ and $\bar{V}$. The image stress field, normalized by the FrankRead stress, acting along the bow-out dislocation position, $(y(x)+2 h / 3) / L_{y}$ as predicted by the analysis (11a), (11b) and the $3 d$ curved image array is shown in Figs. 2a,b for edge and screw dislocations, respectively, for several different normalized volumes $\left(\bar{V}=L_{y} L_{z} / L_{x}^{2}\right)$. Agreement between the fully numerical and analytical results is very good along the entire line in all except the most extreme cases, e.g. $\bar{V}<0.3$. While the image stress field generated by the curved dislocation array is complex, the $2 \mathrm{~d}$-model captures the essential physics, scaling, and magnitude of the image fields. Both analyses also demonstrate that the magnitude of the image stresses is largest at the pinning points (positive, acting to push the dislocation outward) and near the peak (negative, acting to oppose increased bow-out).

We verify the agreement between the $3 \mathrm{~d}$ curved image array and the 
This is an author-created, un-copyedited version of an article accepted for publication/published in Modelling And Simulation In Materials Science And Engineering. IOP Publishing Ltd is not responsible for any errors or omissions in this version of the manuscript or any version derived from it. The Version of Record is available online at http://dx.doi.org/10.1088/0965-0393/23/2/025008. Citation details: Szajewski, B. A.; Curtin, W. A. Modelling And Simulation In Materials Science And Engineering 2015, 23, .

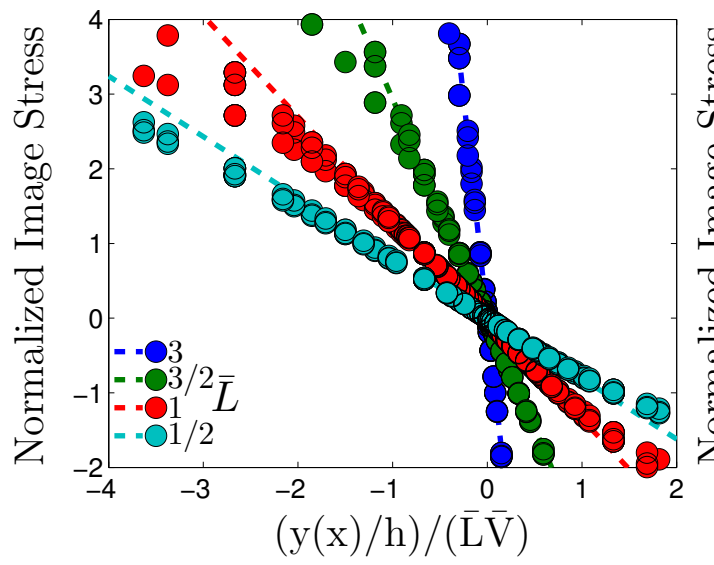

(a) Edge

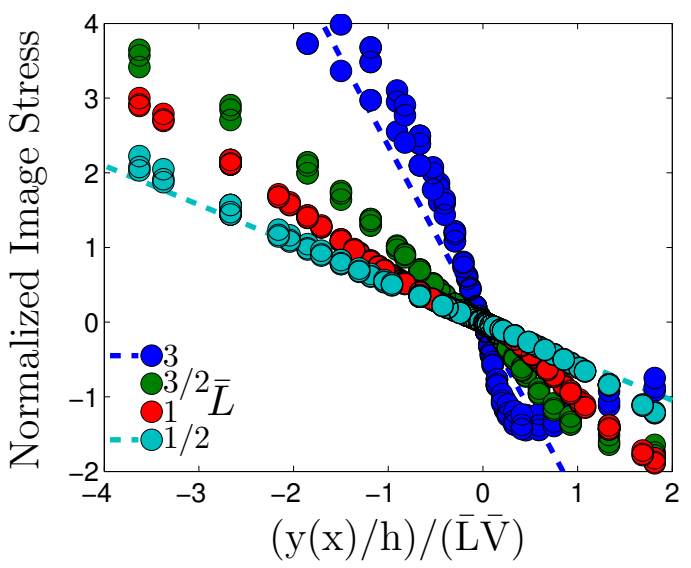

(b) Screw

Figure 3. (a) Edge and (b) Screw. Normalized image stress (image stress field, $\tau_{i m g}[y(x)]$, divided by Frank-Read stress, $\mu b / L_{x}$, and extent of bow-out, $\left.h / L_{x}\right)$ vs. ratio of the normalized position along the dislocation line, $y(x) / h$, to the product of the normalized volume and aspect ratio, $\bar{V} \bar{L}$, (i.e. $(\mathrm{y}(\mathrm{x}) / \mathrm{h}) /(\bar{L} \bar{V}))$ for several aspect ratios as predicted by the $3 \mathrm{~d}$ discrete analysis (symbols) and by the $2 \mathrm{~d}$ analysis (dashed lines) for the peak $(y=h / 3)$ and pinning point $(y=-2 h / 3)$.

$2 \mathrm{~d}$ analysis more generally in the following manner. The image stresses at position $(\mathrm{x}, \mathrm{y}, 0)$ are uniquely specified by the $y$ coordinate since $\mathrm{x}$ is a function of $\mathrm{y}$. We therefore use a normalized position $y / h$ ranging from $-2 / 3$ at the pinning points $\left(x=+/-L_{x} / 2\right)$ to $+1 / 3$ at the point of peak bow-out $(x=0)$ in accordance with Fig. 1d. The scaling analysis, Eqs. (11) and (12), suggests that the image stress field is proportional to both the Frank-Read stress, $\mu b / L_{x}$ and the extent of bow-out, $h / L_{x}$, and scales inversely with the normalized volume $\left(\bar{V}=L_{y} L_{z} / L_{x}^{2}\right)$. Figures $3 \mathrm{a}$,b show the normalized image stress fields $\left(\tau_{i m g}[y(x)] /\left[\left(\mu b / L_{x}\right)\left(h / L_{x}\right)\right]\right)$ at the two extremities of the bow-out (i.e. the peak: $(x=0, y=h / 3)$ and the pinning points: $\left.\left(x=+/-L_{x} / 2, y=-2 h / 3\right)\right)$ for a wide range of cell aspect ratios $\left(\bar{L}=L_{y} / L_{z}\right)$, normalized volumes $\left(\bar{V}=L_{y} L_{z} / L_{x}^{2}\right)$, and extents of bow-out $\left(h / L_{x}\right)$, (i) as computed numerically (symbols) and (ii) as predicted by the analytic model (dashed lines) for the entire bow-out $\left(-2 / 3 \leq \frac{y(x)}{h} \leq 1 / 3\right)$. In general, the results for different amounts of bow-out $h$ collapse very well using this normalization. The analytic model and the numerical model agree quite well where $h / L_{y}<<1$; at larger $h / L_{y}$ the neglected curvature in the $2 \mathrm{~d}$ analytic model becomes non-negligible.

Having validated the $2 \mathrm{~d}$ analysis against the $3 \mathrm{~d}$ analysis, we now note 
This is an author-created, un-copyedited version of an article accepted for publication/published in Modelling And Simulation In Materials Science And Engineering. IOP Publishing Ltd is not responsible for any errors or omissions in this version of the manuscript or any version derived from it. The Version of Record is available online at http://dx.doi.org/10.1088/0965-0393/23/2/025008. Citation details: Szajewski, B. A.; Curtin, W. A. Modelling And Simulation In Materials Science And Engineering 2015, 23, .

several predicted scalings that emerge from the 2d analysis (Eqs. 11a,b and Eqs. 16a,b). Both Eqs. 11a,b and 16a,b show that the effects of cell simulation dimensions are contained in the quantities $\bar{\tau}_{i m g}^{\text {edge }}$ and $\bar{\tau}_{\text {img }}^{\text {screw }}$. Figure 4 thus shows these quantities, multiplied by the normalized volume $\bar{V}$, as a function of $\bar{L}$. As expected, for larger overall cells, the magnitude of the image stress decreases. However, there are wide variations in the trend as a function of the aspect ratio $(\bar{L})$. Therefore, the choice of simulation cell aspect ratio and normalized volume can have a significant affect on the apparent stresses and thus both simulation cell volume and shape must be chosen carefully. Other trends emerging from the analysis are that:

1. For a fixed nominal dislocation line length $L_{x}$, and fixed aspect ratio $\bar{L}=L_{y} / L_{z}$, the normalized image stress scales inversely with the simulation volume.

2. For an initial dislocation line length $L_{x}$ and fixed volume $V$, the normalized image stress depends only on $\bar{L}$.

3. With varying length $L_{x}$, the normalized image stress is constant for constant normalized volume $\bar{V}$ and constant aspect ratio $\bar{L}$; in other words, increasing the overall system size proportionally has no affect on the normalized image stress.

4. For a given volume $\bar{V}$ there is an optimal aspect ratio $\bar{L}$ to minimize the image stresses independent of $L_{x}$, with $\bar{L}_{\text {opt }} \approx 0.8$ for the edge and $\bar{L}_{\text {opt }} \approx \sqrt{2}$ for the screw.

5. Since a bow-out of $h$ scales with the applied stress as $\tau_{a p p} \sim\left(\mu b / L_{x}\right)\left(h / L_{x}\right)$, the effective image stress scales with the true applied stress, i.e. $\tau_{i m g, e f f} / \tau_{\text {app }}$ remains constant, independent of the applied stress.

\section{Discussion \& Application}

Our results show that the effective normalized image stress is approximately quadratic in $L_{x}$ because of the inverse relationship with the normalized volume $\left(\frac{\tau_{i m g, e f f}}{\left(\frac{\mu b}{L_{x}}\right)\left(\frac{h}{L_{x}}\right)} \sim \bar{V}^{-1} \sim L_{x}^{2}\right)$. Thus, a typical study in which $L_{y}$ and $L_{z}$ are fixed and the length $L_{x}$ is varied to study the effects of obstacle spacing can lead to large variations in image stresses that contaminate the intended measurements. Fig. 4 shows a large difference in the dimensionless image stress gradient, Eqs. (12a) and (12b) between a nominally edge and screw dislocation PAD simulation. The dimensionless image stress gradient for the screw dislocation is much smaller than that for the edge e.g. $\left.\frac{\overline{\bar{i}}_{\text {img }}^{\text {screw }}}{\bar{\tau}_{\text {img }}^{\text {edge }}}\right|_{\bar{L}=1} \approx \frac{1}{3}$. 
This is an author-created, un-copyedited version of an article accepted for publication/published in Modelling And Simulation In Materials Science And Engineering. IOP Publishing Ltd is not responsible for any errors or omissions in this version of the manuscript or any version derived from it. The Version of Record is available online at http://dx.doi.org/10.1088/0965-0393/23/2/025008. Citation details: Szajewski, B. A.; Curtin, W. A. Modelling And Simulation In Materials Science And Engineering 2015, 23, .

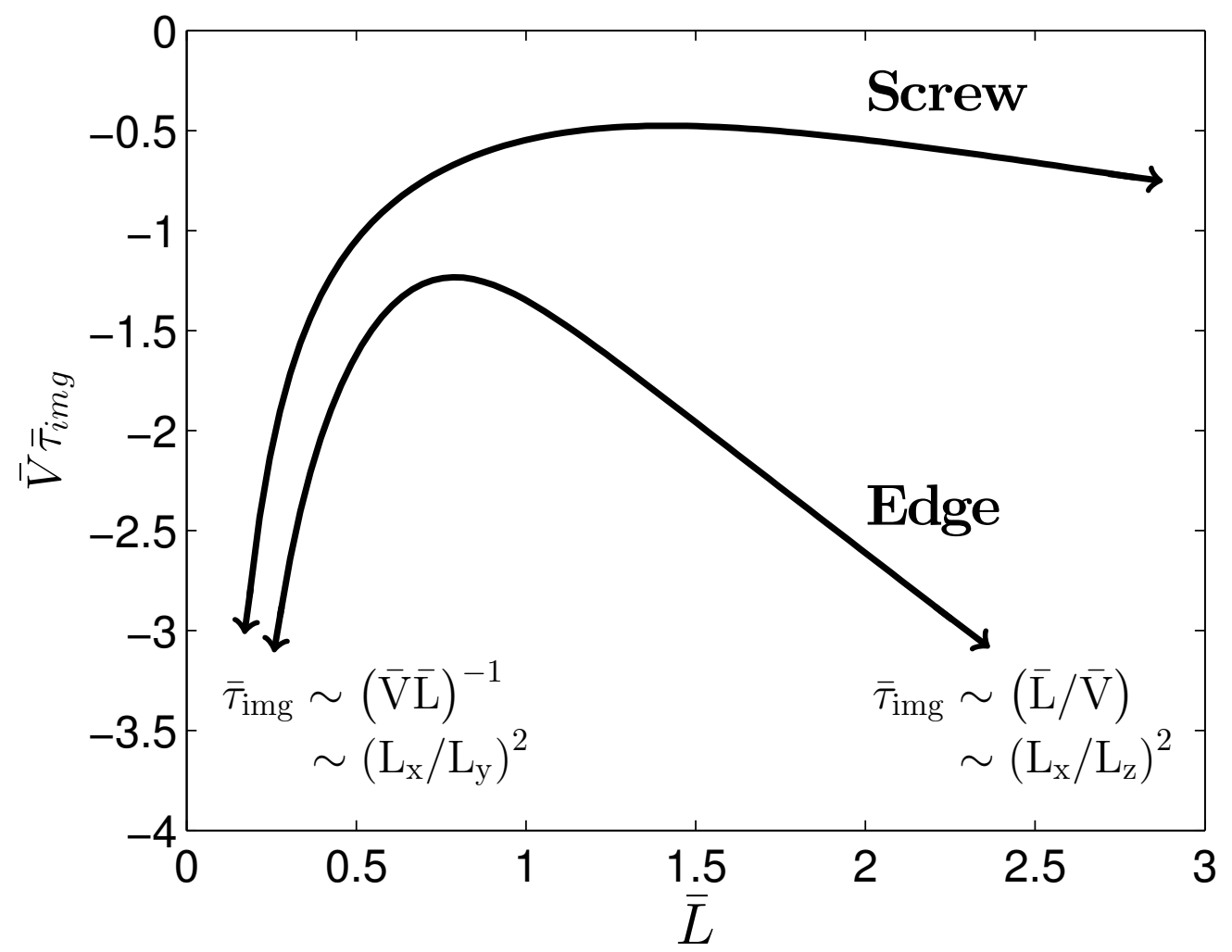

Figure 4. Dimensionless image stress gradient $\left(\bar{\tau}_{i m g}\right)$ times normalized volume $\left(\bar{V}=\frac{L_{y} L_{z}}{L_{x} L_{x}}\right)$ vs. aspect ratio $\left(\bar{L}=\frac{L_{y}}{L_{z}}\right)$ for the nominally edge and screw dislocation. The asymptotic behavior of $\bar{\tau}_{i m g}$ for $\bar{L}>>1$ and $\bar{L}<<1$ is shown to be $\sim 1 / L_{z}^{2}$ and $\sim 1 / L_{y}^{2}$ respectively. The optimal aspect ratio which minimizes spurious image stress for the nominally edge dislocation is $\bar{L}_{\text {opt }} \approx 0.8$ and for the nominally screw dislocation is $\bar{L}_{\text {opt }} \approx \sqrt{2}$. 
This is an author-created, un-copyedited version of an article accepted for publication/published in Modelling And Simulation In Materials Science And Engineering. IOP Publishing Ltd is not responsible for any errors or omissions in this version of the manuscript or any version derived from it. The Version of Record is available online at http://dx.doi.org/10.1088/0965-0393/23/2/025008. Citation details: Szajewski, B. A.; Curtin, W. A. Modelling And Simulation In Materials Science And Engineering 2015, 23, .

Furthermore, at a fixed applied stress, a nominally edge dislocation is expected to bow-out more than a screw dislocation at the same applied stress due to the reduced line tension making the image stress, relative to the applied stress, much larger for the edge dislocation. For the nominally edge dislocation both the increased dimensionless image stress gradient and the increased bow-out at a fixed applied stress suggest that the image stress relative to the applied stress is generally much greater for the nominally edge dislocation. With all of these subtleties, extracting quantitative results from parametric studies requires a sufficiently large minimum cell volume as to mitigate spurious image type effects all together.

We now demonstrate, through example, the interpretation and usage of Fig. 4 as a guide to the design of studies involving dislocation/obstacle interactions. We examine a typical simulation of a nominally edge dislocation impinging on obstacles with spacing $L_{x}=50 \mathrm{~nm}$ in $\mathrm{Al}$ (FCC, lattice constant $\mathrm{a}=4.03 \stackrel{\circ}{\mathrm{A}}$, atomic density $\left.=61 \frac{\mathrm{atoms}}{n \mathrm{~m}^{3}}\right)$. We start by considering equal glide and out-of-plane periodic lengths, $L_{y}=L_{z}=40 \mathrm{~nm}$ for simplicity. These dimensions lead to $\bar{V}=L_{y} L_{z} / L_{x}^{2}=0.64$ and $\bar{L}=L_{y} / L_{z}=1$. The number of atoms required for this simulation follows as $N=\left(L_{x}^{3}\right)(\bar{V})\left(61 / n m^{3}\right) \approx 4.9$ million atoms. One or a few calculations at this size are computationally feasible, however numerous computations of this size quickly become computationally expensive. From Fig. 4, we find that $\bar{\tau}_{\text {img }}^{\text {edge }} \approx-1.4 / 0.64=-2.19$ and the effective image stress is thus $\tau_{\text {img,eff }}=2.19\left(\mu b / L_{x}\right)\left(4 h / 45 L_{x}\right)$ at bow-out $h$. If this magnitude is acceptable, we might wish to use half the number of atoms and the same aspect ratio $\bar{L}=1$, or $L_{y}=L_{z}=28.3 \mathrm{~nm}$. The effective image stress would then double because the volume is halved at fixed $\bar{L}$. But if instead we halved the number of atoms by changing the aspect ratio to $\bar{L}=0.8$ with $L_{y}=25.3 \mathrm{~nm}, L_{z}=31.6 \mathrm{~nm}$, and thus $\bar{V}=0.32$, we obtain an effective image stress of $\tau_{\text {img,eff }}=3.91\left(\mu b / L_{x}\right)(4 h / 45)$ which is less than twice the original value.

To further validate our analysis quantitatively, we now compare results in a typical PAD simluation and in a multiscale image-free simulation [45] of the same problem: bow-out of a pinned nominally edge dislocation under an applied stress. The studies were performed on $\mathrm{Al}$ using the ErcolessiAdams [53] EAM potential having material properties $\mu=30.8 \mathrm{GPa},|\mathbf{b}|$ $=2.85 \AA$ and $\nu=0.35$, with an obstacle spacing of $L_{x}=50 \mathrm{~nm}$. The PAD simulation, reported in [54], used dimensions $L_{y}=L_{z}=20 \mathrm{~nm}$. The multiscale image-free simulation was performed using the Coupled Atomistic/Discrete Dislocation (CADD) method [45, 55], in which an atomistic domain is embedded in a fully anisotropic continuum elastic domain with a seamless atom/continuum interface that introduces no spurious forces. The multiscale simulation corresponds effectively to infinite dimensions $L_{y}, L_{z}$. In 
This is an author-created, un-copyedited version of an article accepted for publication/published in Modelling And Simulation In Materials Science And Engineering. IOP Publishing Ltd is not responsible for any errors or omissions in this version of the manuscript or any version derived from it. The Version of Record is available online at http://dx.doi.org/10.1088/0965-0393/23/2/025008. Citation details: Szajewski, B. A.; Curtin, W. A. Modelling And Simulation In Materials Science And Engineering 2015, 23, .

Fig. 5, we show the equilibrium configurations of three curved dislocation structures: two using the traction PAD method [54] under a PAD simulation applied stresses of $\sim 30 \mathrm{MPa}$ and $\sim 45 \mathrm{MPa}$ and one using the multiscale image-free method [45] under a (true) applied stress of $\sim 30 \mathrm{MPa}$. The traction PAD simulation at $\sim 45 \mathrm{MPa}$ closesly matches the image-free result at $\sim 30 \mathrm{MPa}$, indicating that the image stresses correspond to an effective applied stress of $\sim 15 \mathrm{MPa}$, one-half of the true applied value. The traction PAD simulation at a simulation stress equal to that in the multiscale simulation, $\sim 30 \mathrm{MPa}$, exhibits significantly less bow-out; in fact, the peak bow-out is approximately $2 / 3$ that of the traction PAD simulation at $3 / 2$ larger applied stress, as expected since both the bow-out and the effective image stress scale with the applied stress. We now use our results to predict the effective image stress. For this PAD simulation cell, $\bar{L}=1$ and $\bar{V}=0.16$. From Fig. 4, we find $\bar{\tau}_{i m g}^{\text {edge }}=-(1.4 / 0.16)$ and thus from Eq. 16a $\tau_{\text {img,eff }}^{\text {edge }}=(1.4 / 0.16)\left(\mu b / L_{x}\right)\left(4 h / 45 L_{x}\right)$. From Fig. $5, h / L_{x} \approx 0.14$ and so $\tau_{\text {img,eff }}^{\text {edge }}=18.4 \mathrm{MPa}$. The predicted effective image stress agrees very well with the measured image stress, with an error of $\approx 25 \%$. The over-estimate for this particular case is consistent with Fig. 2a, where the analytic $2 \mathrm{~d}$ result (dashed blue line) over-estimates the numerical 3d result (symbols) for $\bar{V}=0.16$.

Finally, we extend the analyses to several dislocation/obstacle simulation studies available within the literature. In Table 1 we report the absolute dislocation line length $L_{x}$, aspect ratio $(\bar{L})$, and normalized volume $(\bar{V})$ reported for various simulation cells. For these values, we compute the normalized effective image stress $\left(\tau_{\text {img,eff }} /\left[\left(\frac{\mu b}{L_{x}}\right)\left(\frac{4 h}{45 L_{x}}\right)\right]\right)$ for studies involving nominally edge and screw dislocations. We note that the normalized effective image stress for the PAD depicted in Fig. 5 corresponds to the value $\sim 8.2$ which corresponded to an error of $\approx 50 \%$ (45 MPa vs. $30 \mathrm{MPa}$ ) in the applied stress. With the exception of the simulation reported by Cheng and coworkers [1], all of the other edge studies have effective image stresses that are comparable to or worse than the result in Fig. 5. Osetsky [56] and Dong [54] attempted some limited convergence studies by increasing the simulation cell in the in-plane direction $\left(L_{y}\right)$ for a fixed obstacle spacing. As predicted by (12a), however, these studies reproduced nearly the same normalized effective image error while adding computational cost with the approximately doubled volume. Thus, although the coarse results appear to have converged, they still have the same image error embedded in the results! Several similar studies involved "strain" controlled boundary conditions on the top and bottom surfaces, where the dislocation is driven by an imposed displacement rather than an imposed stress. As noted by Rod- 
This is an author-created, un-copyedited version of an article accepted for publication/published in Modelling And Simulation In Materials Science And Engineering. IOP Publishing Ltd is not responsible for any errors or omissions in this version of the manuscript or any version derived from it. The Version of Record is available online at http://dx.doi.org/10.1088/0965-0393/23/2/025008. Citation details: Szajewski, B. A.; Curtin, W. A. Modelling And Simulation In Materials Science And Engineering 2015, 23, .

ney [57], the image stresses for the displacement boundary conditions are expected to be comparable to, but larger than, those for traction control. Our estimates of the effective image stresses are thus probably conservative in these cases. Table 1 also shows the normalized effective image stress for three nominally screw simulation cells [58] but for illustrative purposes only because the actual studies did not involve bow-out type configurations confined to the central slip plane. We conclude with a brief, general discussion about testing the size of simulation cells for converged simulation results. As seen in Table 1 , it is remarkably easy to change a single length of the simulation cell, either the out-of-plane length $L_{z}$ or the periodic glide length $L_{y}$, and recover a "converged" yet wrong result. Examination of Eqs. (12a), (12b) and Fig. 4 provides a quantitative explanation for this, demonstrating that when $\bar{L}>>1$ or $\bar{L}<<1$ changing $L_{y}$ or $L_{z}$, respectively, has virtually no influence on the image stress. Instead, fixing the aspect ratio $(\bar{L})$ to the optimum value and varying the normalized volume $(\bar{V})$ is a more suitable method to test for converged results.

\section{Summary}

We have investigated the nature of undesirable spurious image stresses arising from the bowing out of a dislocation around obstacles in a finite sized atomistic simulation volume, and have provided a general map of the magnitude of the spurious effects as a function of the simulation cell size and shape. We have further demonstrated that, independent of the cell size, these image stresses scale with the nominally applied load. For both a nominally edge and screw dislocation, we have found special aspect ratios $\left(\bar{L}=L_{y} / L_{z}\right)$ which minimize spurious image forces for a given number of atoms (given simulation volume). We have made a direct comparison between a dislocation structure under an applied load in a finite simulation cell and an image-free multiscale simulation [45] of the same problem, showing that our analysis is quantitatively accurate. Finally, we have evaluated the image stresses that would have arisen in several other studies within the literature, predicting comparable errors. Overall, this work provides a clear and simple benchmark for designing atomistic simulations with controlled and minimized image effects in the many physical situations involving dislocation bow-out.

\section{Acknowledgements}

The authors thank the Fonds National Suisse for support of this work through the project 200021_140506 entitled "Coupled Atomistic/DiscreteDislocations in 3d (CADD3d)". 
This is an author-created, un-copyedited version of an article accepted for publication/published in Modelling And Simulation In Materials Science And Engineering. IOP Publishing Ltd is not responsible for any errors or omissions in this version of the manuscript or any version derived from it. The Version of Record is available online at http://dx.doi.org/10.1088/0965-0393/23/2/025008. Citation details: Szajewski, B. A.; Curtin, W. A. Modelling And Simulation In Materials Science And Engineering 2015, 23, .

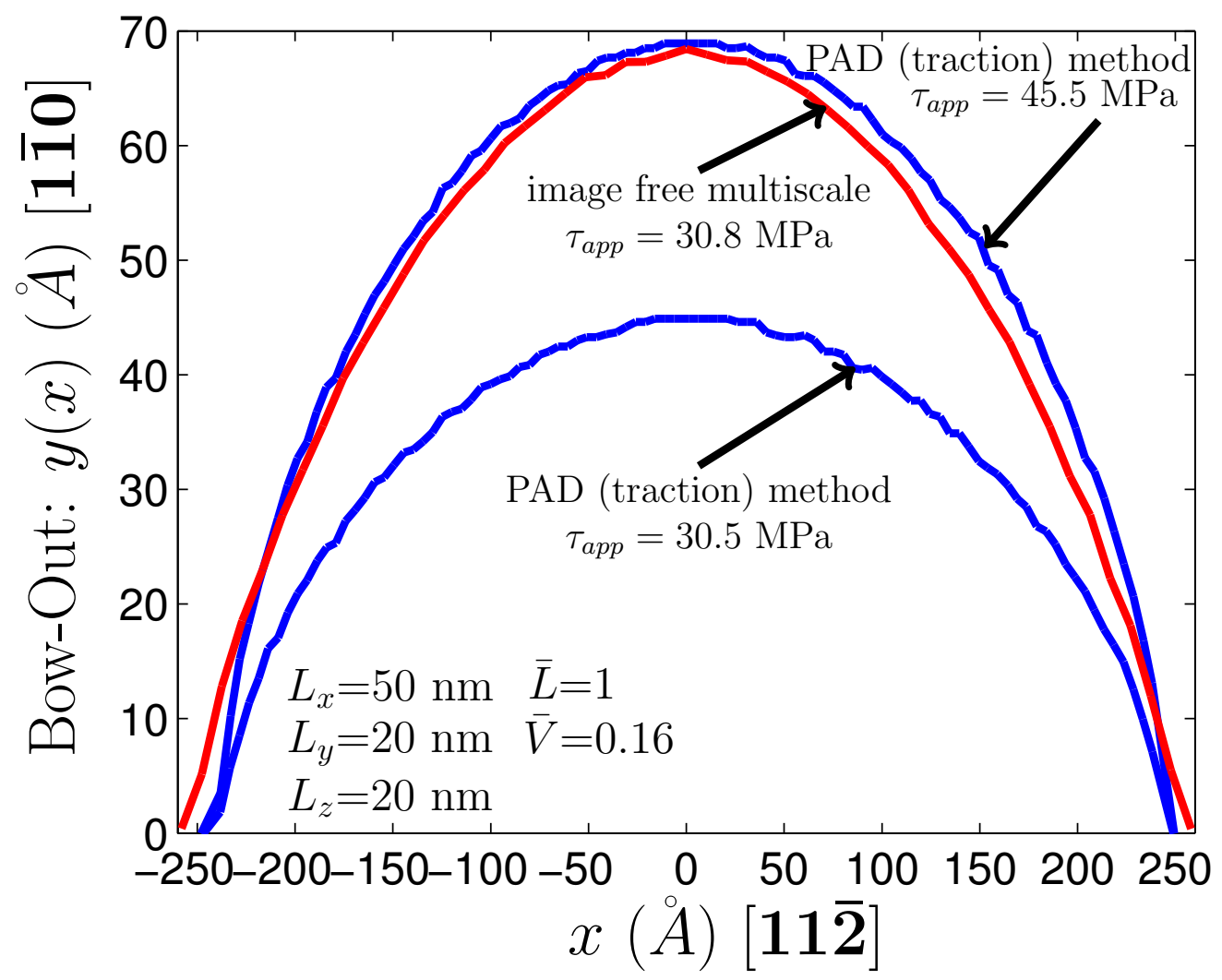

Figure 5. Equilibrium configurations of a nominally edge dislocation bowing out under an applied stress using the traction PAD method [54] (blue) and an image free multiscale method [45] (red). The PAD simulation cell contained an obstacle spacing of $L_{x}=50 \mathrm{~nm}$ with periodicity and free surfaces a distance $L_{y}=L_{z}=20 \mathrm{~nm}$. Two PAD bow-outs are depicted under a nominal applied stress of $\sim 30 \mathrm{MPa}$ and $\sim 45 \mathrm{MPa}$. A single artifact free bow-out is shown at an applied stress of $\sim 30 \mathrm{MPa}$ corresponding with the PAD bow-out of $\sim 45 \mathrm{MPa}$. For this simulation cell geometry, nominal dislocation structure (edge) and Ercolessi-Adams [53] potential: $\frac{\tau_{i m g, e f f}^{\text {edge }}}{\tau_{\text {app }}} \approx 50 \%$. Partial dislocations were detected using a common neighbor analysis and averaged to yield a single, continuous center line for the dislocation bow-out. 
This is an author-created, un-copyedited version of an article accepted for publication/published in Modelling And Simulation In Materials Science And Engineering. IOP Publishing Ltd is not responsible for any errors or omissions in this version of the manuscript or any version derived from it. The Version of Record is available online at http://dx.doi.org/10.1088/0965-0393/23/2/025008. Citation details: Szajewski, B. A.; Curtin, W. A. Modelling And Simulation In Materials Science And Engineering 2015, 23, .

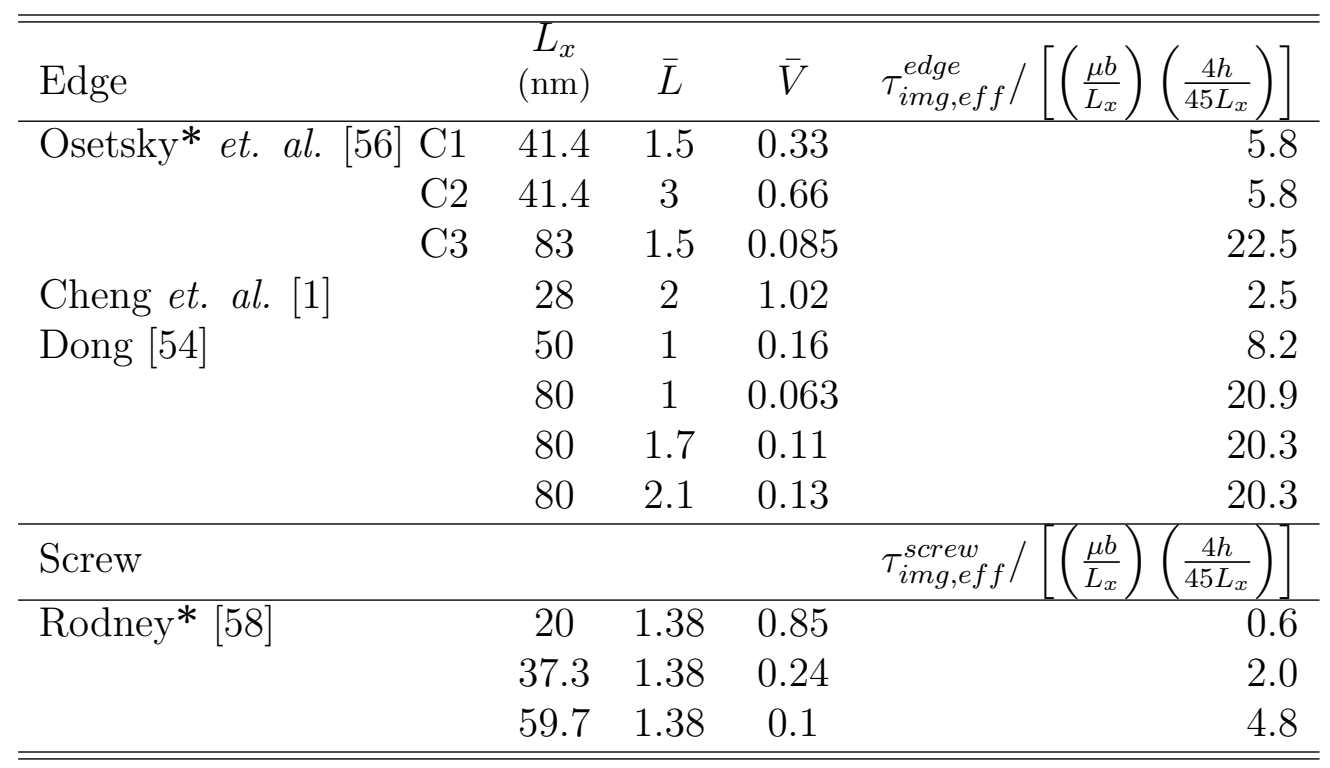

Table 1. Simulation cell obstacle spacing $\left(L_{x}\right)$, aspect ratio $\left(\bar{L}=L_{y} / L_{z}\right)$, normalized volume $\left(\bar{V}=L_{y} L_{z} / L_{x}^{2}\right)$, and resulting normalized effective image stress analysis, Eqs. (16a) and (16b) applied to several dislocation/obstacle interaction studies $(\nu=1 / 3)$. The dimensionless image stress gradient has been calculated directly from (12a) and (12b) for the nominally edge and screw dislocation respectively. When $\bar{L}>>1$ or $\bar{L}<<1$ doubling the simulation volume can result in an identical (and non-negligible) image stress (e.g. Osetsky [56] C1 and C2 and Dong [54]). *These simulations were performed using either a "strain" controlled (Osetsky [56]) or alternatively bounded (Rodney [58]) PAD and so the computation of $\bar{\tau}_{i m g}(\bar{L}, \bar{V})$ remains inexact. As noted by Rodney [57] however, it is expected to be comparable to, but larger in the case of "strain" control due to the mismatch between the curved dislocation structure and straight dislocation field within the PAD region. 
This is an author-created, un-copyedited version of an article accepted for publication/published in Modelling And Simulation In Materials Science And Engineering. IOP Publishing Ltd is not responsible for any errors or omissions in this version of the manuscript or any version derived from it. The Version of Record is available online at http://dx.doi.org/10.1088/0965-0393/23/2/025008. Citation details: Szajewski, B. A.; Curtin, W. A. Modelling And Simulation In Materials Science And Engineering 2015, 23, .

\section{References}

[1] Cheng Y, Bitzek E, Weygand D, and Gumbsch P. Atomistic simulation of dislocation-void interactions under cyclic loading. Modelling and Simulation in Materials Science and Engineering, 18:025006, 2010.

[2] Hatano T and Matsui H. Molecular dynamics investigation of dislocation pinning by a nanovoid in copper. Physical Review B, 72:094105, 2005.

[3] Robach JS, Robertson IM, Lee HJ, and Wirth BD. Dynamic observations and atomistic simulations of dislocation-defect interactions in rapidly quenched copper and gold. Acta Materialia, 54:1679-1690, 2006.

[4] Picu RC and Zhang D. Atomistic study of pipe diffusion in al-mg alloys. Acta Materialia, 52:161-171, 2004.

[5] Schmauder S and Kohler C. Atomistic simulations of solid solution strengthening of $\alpha$-iron. Computational Materials Science, 50:1238$1243,2011$.

[6] Olmsted DL, Hector Jr. LG, and Curtin WA. Molecular dynamics study of solute strengthening in al/mg alloys. Journal of the Mechanics and Physics of Solids, 54:1763-1788, 2006.

[7] Velasco M, Van Swygenhoven H, and Brandl C. Coupled grain boundary motion in a nanocrystalline grain boundary network. Scripta Materialia, $65: 151-154,2011$.

[8] Farkas D, Froseth AG, and Van Swygenhoven H. Grain boundary migration during room temperature deformation of nanocrystalline Ni. Scripta Materialia, 55:695 - 698, 2006.

[9] Hasnaoui A, Derlet PM, and Van Swygenhoven H. Interaction between dislocations and grain boundaries under an indenter - a molecular dynamics simulation. Acta Materialia, 52:2251 - 2258, 2004.

[10] Rao S, Parthasarathy TA, and Woodward C. Atomistic simulation of cross-slip processes in model fcc structures. Philosophical Magazine, 79:1167 - 1192, 1999 .

[11] Bitzek E, Brandl C, Derlet PM, and Van Swygenhoven H. Dislocation cross-slip in nanocrystalline fcc metals. Physical Review Letters, 100:235501 - 235505, 2008. 
This is an author-created, un-copyedited version of an article accepted for publication/published in Modelling And Simulation In Materials Science And Engineering. IOP Publishing Ltd is not responsible for any errors or omissions in this version of the manuscript or any version derived from it. The Version of Record is available online at http://dx.doi.org/10.1088/0965-0393/23/2/025008. Citation details: Szajewski, B. A.; Curtin, W. A. Modelling And Simulation In Materials Science And Engineering 2015, 23, .

[12] Bitzek E, Derlet PM, and Van Swygenhoven H. Dislocation cross-slip in nanocrystalline fcc metals. Physical Review Letters, 100:235501, 2008.

[13] Warner DH, Curtin WA, and Qu S. Rate dependence of crack-tip processes predicts twinning trends in fcc metals. Nature Materials, 6:876 881, 2007.

[14] Zhang Y, Liu XY, Millett PC, Tonks M, Andersson DA, and Biner B. Crack tip plasticity in single crystal $\mathrm{uo}_{2}$ : Atomistic simulations. Journal of Nuclear Materials, 430:96 - 105, 2012.

[15] Patinet S and Proville L. Dislocation pinning by substitutional impurities in an atomic-scale model for $\mathrm{Al}(\mathrm{Mg})$ solid solutions. Philosophical Magazine, 91:1581-1606, 2011.

[16] Proville L, Rodney D, Brechet Y, and Martin G. Atomic-scale study of dislocation glide in a model solid solution. Philosophical Magazine, 86:3893 - 3920, 2006.

[17] Bacon DJ, Osetsky YN, and Rodney D. Dislocation-obstacle interactions at the atomic level. Dislocations in Solids, 15:1-90, 2009.

[18] Tapasa K, Osetsky YN, and Bacon DJ. Computer simulation of interaction of an edge dislocation with a carbon interstitial in $\alpha$-iron and effects on glide. Acta Materialia, 55:93-104, 2007.

[19] Monnet G. Mechanical and energetical analysis of molecular dynamics simulations of dislocation-defect interactions. Acta Materialia, 55:50815088, 2007.

[20] Rodney D and Martin G. Dislocation pinning by glissile interstitial loops in a nickel crystal: A molecular-dynamics study. Physical Review $B, 61: 8714-8725,2000$.

[21] Osetsky YN, Bacon DJ, and Mohles V. Atomic modelling of strengthening mechanisms due to voids and copper precipitates in $\alpha$-iron. Philosophical Magazine, 83:3623-3641, 2003.

[22] Osetsky YN and Bacon DJ. An atomic-level model for studying the dynamics of edge dislocations in metals. Modelling and Simulation in Materials Science and Engineering, 11:427-446, 2003.

[23] Osetsky YN and Bacon DJ. Comparison of void strengthening in fcc and bcc metals: Large-scale atomic-level modelling. Materials Science \& Engineering A, 400-401:374-377, 2005. 
This is an author-created, un-copyedited version of an article accepted for publication/published in Modelling And Simulation In Materials Science And Engineering. IOP Publishing Ltd is not responsible for any errors or omissions in this version of the manuscript or any version derived from it. The Version of Record is available online at http://dx.doi.org/10.1088/0965-0393/23/2/025008. Citation details: Szajewski, B. A.; Curtin, W. A. Modelling And Simulation In Materials Science And Engineering 2015, 23, .

[24] Madec R, Devincre B, Kubin L, Hoc T, and Rodney D. The role of collinear interaction in dislocation-induced hardening. Science, 301:1879 $-1882,2003$.

[25] Xiong L, Xu S, McDowell D, and Chen Y. Concurrent atomisticcontinuum simulations of dislocation-void interactions in fcc crystals. International Journal of Plasticity, 65:33 - 42, 2015.

[26] Shim J-H, Cho YW, Kwon SC, Kim WW, and Wirth BD. Screw dislocation assisted martensitic transformation of a bcc $\mathrm{Cu}$ precipitate in bcc Fe. Applied Physics Letters, 90:021906, 2007.

[27] Shim J-H, Kim D-I, Jung W-S, Cho YW, and Wirth BD. Strengthening of nanosized bcc $\mathrm{Cu}$ precipitate in bcc Fe: A molecular dynamics study. Materials Transactions, 50:2229 - 2234, 2009.

[28] Lee H-J and Wirth BD. Molecular dynamics simulation of the interaction between a mixed dislocation and a stacking fault tetrahedron. Philosophical Magazine, 89:821 - 841, 2009.

[29] Khater HA, Monnet G, Terentyev D, and Serra A. Dislocation glide in Fe-carbon solid solution: From atomistic to continuum level description. International Journal of Plasticity, 62:34 - 49, 2014.

[30] Rong Z, Bacon DJ, and Osetsky YN. Dynamics of drag of self-interstitial clusters by an edge dislocation in iron. Materials Science and Engineering: A, 400-401:378 - 381, 2005.

[31] Bitzek E and Gumbsch P. Atomistic study of drag, surface and intertial effects on edge dislocation in face-centered cubic materials. Materials Science and Engineering: A, 387 - 389:11 - 15, 2004.

[32] Bitzek E and Gumbsch P. Dynamic aspects of dislocation motion: Atomistic simulations. Materials Science and Engineering: A, 400 - 401:40 44, 2005.

[33] Domain C and Monnet G. Simulation of screw dislocation motion in iron by molecular dynamics simulations. Physical Review Letters, 95:215506, 2005.

[34] Needleman A and van der Giessen E. Discrete dislocation plasticity: A simple planar model. Modelling and Simulation in Materials Science and Engineering, 3:689 - 735, 1995. 
This is an author-created, un-copyedited version of an article accepted for publication/published in Modelling And Simulation In Materials Science And Engineering. IOP Publishing Ltd is not responsible for any errors or omissions in this version of the manuscript or any version derived from it. The Version of Record is available online at http://dx.doi.org/10.1088/0965-0393/23/2/025008. Citation details: Szajewski, B. A.; Curtin, W. A. Modelling And Simulation In Materials Science And Engineering 2015, 23, .

[35] Weinberger CR, Aubry S, Lee SW, Nix WD, and Cai W. Modelling dislocations in a free-standing thin film. Modelling and Simulation in Materials Science and Engineering, 17:075007, 2009.

[36] Barber JR. Elasticity. Kluwer, $2^{\text {nd }}$ edition, 2002.

[37] Weinberger CR and Cai W. Computing image stress in an elastic cylinder. Journal of The Mechanics and Physics of Solids, 55:2027 - 2054, 2007.

[38] Eshelby JD. Screw dislocations in thin rods. Journal of Applied Physics, $24: 176-179,1953$.

[39] Daw MS, Baskes MI, Bisson CL, and Wolfer WG. Conf. modelling enviornmental effects on crack growth processes. In Jones RH Gerberich WW (Metallurgical Society Warrendale PA 1986), editor, Conf. Modelling Enviornmental Effects on Crack Growth Processes, pages 99 - 124, 1986.

[40] Daw MS, Foiles SM, and Baskes MI. The embedded-atom method: a review of theory and applications. Materials Science Reports, 9:251 310, 1993.

[41] JR Beeler. Radiation Effects Computer Experiments, volume 13. Amsterdam: North-Holland Publishing Company, 1983.

[42] Bitzek E, Koskinen P, Gahler F, Moseler M, and Gumbsch P. Structural relaxation made simple. Physical Review Letters, 97:170201, 2006.

[43] Plimpton S. Fast parallel algorithms for short-range moleculardynamics. Journal of Computational Physics, 117:1 - 19, 1995.

[44] Tang Y and El-Awady A. Atomistic simulations of the interactions of hydrogen with dislocations in fcc metals. Physical Review B, 86:174102, 2012 .

[45] Pavia F and Curtin WA. A parallel multiscale algorithm for 3d concurrent continuum/atomic simulations using lammps. Submitted to: Modelling and Simulation in Materials Science and Engineering, 2014.

[46] Hirth JP and Lothe J. Theory of Dislocations. Wiley, 1982.

[47] Weygand D, Friedman LH, Van der Giessen E, and Needleman A. Aspects of boundary-value problem solutions with three dimensional dislocation dynamics. Modelling and Simulation in Materials Science and Engineering, 10:437 - 468, 2002. 
This is an author-created, un-copyedited version of an article accepted for publication/published in Modelling And Simulation In Materials Science And Engineering. IOP Publishing Ltd is not responsible for any errors or omissions in this version of the manuscript or any version derived from it. The Version of Record is available online at http://dx.doi.org/10.1088/0965-0393/23/2/025008. Citation details: Szajewski, B. A.; Curtin, W. A. Modelling And Simulation In Materials Science And Engineering 2015, 23, .

[48] Chou YT. Screw dislocation arrays in a plate. Journal of Applied Physics, 37:796 - 797, 1966.

[49] Nabarro FR and Kostlan EJ. The stress field of a dislocation lying in a plate. Journal of Applied Physics, 49:5445 - 5448, 1978.

[50] Arsenlis A, Cai W, Tang M, Rhee M, Oppelstrup T, Hommes G, Pierce TG, and Bulatov VV. Enabling strain hardening simulations with dislocation dynamics. Modelling and Simulation in Materials Science and Engineering, 15:553 - 595, 2007.

[51] Kuykendall WP and Cai W. Conditional convergence in 2-dimensional dislocation dynamics. Modelling and Simulation in Materials Science and Engineering, 21:055003, 2013.

[52] Cai W, Bulatov VV, Chang J, Li J, and Yip S. Periodic image effects in dislocation modelling. Philosophical Magazine, 83:539 - 567, 2003.

[53] Ercolessi $\mathrm{F}$ and Adams JB. Interatomic potentials from first-principles calculations: The force-matching method. Europhysics Letters, 26:583 - 588, 1994.

[54] Dong Y. Coupled Dislocation/Dislocation and Solute Strengthening Mechanisms in Metal Alloys. PhD thesis, Brown University, 2013.

[55] Shilkrot LE, Miller RE, and Curtin WA. Coupled atomistic and discrete dislocation plasticity. Physical Review Letters, 89:025501, 2002.

[56] Osetsky YN, Bacon DJ, and Mohles V. Atomic modelling of strengthening mecanisms due to voids and copper precipitates in $\alpha$-iron. Philosophical Magazine, 83:3623 - 3641, 2003.

[57] Rodney D. Activation enthalpy for kink-pair nucleation on dislocations: Comparison between static and dynamic atomic-scale simulations. Physical Review B, 76:144108, 2007.

[58] Rodney D. Molecular dynamics simulation of screw dislocations interacting with interstitital frank loops in a model fcc crystal. Acta Materialia, 52:607-614, 2004. 